Revista Científica de FAREM-Estelí

Medio ambiente, tecnología y desarrollo humano

Año 10 | Núm. 39| Julio-septiembre, 2021

ISSN: 2305-5790

https://rcientificaesteli.unan.edu.ni

DOI: https://doi.org/10.5377/farem.v10i39.12613

\section{Experiencias de duelo en cuidadores de personas con diagnóstico de parálisis cerebral}

\section{Experiences of mourning in caregivers of people diagnosed with cerebral palsy}

\section{Fernanda Vanessa Palacios Escoto}

Egresada de la carrera de Psicología. Universidad Nacional Autónoma de

Nicaragua UNAN Managua. Facultad Regional Multidisciplinaria de Estelí. FAREM Estelí.

Palaciosfergy@gmail.com

\section{José Enrique Ortega Laguna}

Egresado de la carrera de Psicología. Universidad Nacional Autónoma de Nicaragua UNAN Managua. Facultad Regional Multidisciplinaria de Estelí. FAREM Estelí.

joseortegalaguna66@gmail.com

\section{Franklin Jesús Solís Zúniga}

Asesor y docente de la carrera de Psicología. Universidad Nacional Autónoma de Nicaragua UNAN Managua. Facultad Regional Multidisciplinaria de Estelí. FAREM Estelí.

frankzuni@gmail.com

\section{RESUMEN}

El duelo es un proceso natural que atraviesan las personas como reacción ante la pérdida de una persona o algo significativo, se tiende a pensar en duelo solo en el contexto de la muerte de una persona querida, pero también puede producirse como reacción ante la pérdida del ser anhelado e idealizado, en este caso se produce ante el diagnóstico de discapacidad de una persona en el seno familiar. La investigación se realizó con el objetivo de comprender las experiencias de duelo en cuidadoras de personas con diagnóstico de parálisis cerebral, en el Centro Los Pipitos Matagalpa, agosto - diciembre 2020. Se llevó a cabo a través de un enfoque cualitativo diseño fenomenológico y alcance exploratorio. La muestra estuvo conformada por cuatro cuidadoras afiliadas al centro los Pipitos de la ciudad de Matagalpa, seleccionadas mediante un muestreo por conveniencia. Los instrumentos aplicados fueron entrevistas en profundidad a las cuidadoras, entrevista semiestructurada a una psicóloga que trabaja el área de salud mental, guía de observación a las cuidadoras. Los principales resultados demuestran la existencia de un proceso doloroso en las cuidadoras de personas con diagnóstico de parálisis cerebral, incluso una de las cuidadoras presenta un duelo aún no resuelto. Se percibió mucho estrés, sobre todo el apego y sobreprotección que tienen a sus familiares. Finalmente, se plantea un plan de intervención general con estrategias y técnicas de intervención psicológica para afrontar el duelo en cuidadores de personas con Parálisis Cerebral. (C) 2021 - Revista Científica de FAREM-Estelí.

(c) (i) (2) Este trabajo está licenciado bajo una Licencia Internacional Creative Commons 4.0 Atribución-NoComercial-Compartirlgual.
RECIBIDO

$14 / 05 / 2021$

ACEPTADO

$27 / 09 / 2021$

\section{PALABRAS CLAVE}

Experiencias de duelo; cuidadoras; Parálisis Cerebral; repercusiones psicológicas; estrategias de intervención. 


\section{ABSTRACT}

Mourning is a natural process that people go through as a reaction to the loss of a person or something significant, we tend to think of mourning only in the context of the death of a love one, but it can also occur as a reaction to the loss of a longed-for and idealized being, in this case it occurs when a person in the diagnosed with a disability. The research was conducted with the objective of understanding the mourning experiences of caregivers of people diagnosed with cerebral palsy at the Los Pipitos Matagalpa Center, august - december 2020. It was carried out through qualitative approach phenomenological design and exploratory research. The sample consisted of four caregivers affiliated with the Los Pipitos center in the city of Matagalpa, selected thought convenience sampling. The instruments applied were in-depth interview with the caregivers, a semi-structured interview with a psychologist working in the mental health area, observation guide for the caregivers. The main results show the existence of a painful process in the caregivers of people diagnosed with cerebral palsy, and one of the caregivers even presents a mourning unresolved. A lot of stress perceived, especially the attachment and overprotection they have to their relatives. Finally, a general intervention plan is proposed with strategies and psychological intervention techniques to cope with mourning in caregivers of people with cerebral palsy.

\section{KEYWORDS}

Mourning experiences; caregivers; Cerebral Palsy; psychological repercussions; intervention strategies. 


\section{INTRODUCCIÓN}

El duelo por la ruptura de una imagen idealizada de un niño sano y normal también trae consigo una serie de altibajos emocionales, situación que los individuos deben pasar, para traer consigo la aceptación de realidad de la persona con discapacidad, a lo que se le conoce como Duelo ante la discapacidad de una persona (Olivares Angeles, 2013).

Madrigal (2004, pág. 5), en el informe del Observatorio de la discapacidad señala que "el término Parálisis hace referencia a una debilidad o problema en la utilización de los músculos, que se manifiesta con alteraciones en el control del movimiento, el tono muscular y la postura". Según la caracterización de esta patología la lesión tiene que ocurrir en el período comprendido entre los primeros días de gestación y los tres o cinco años de vida.

El duelo, es el proceso de adaptación que permite restablecer el equilibrio personal y familiar roto por las situaciones de muerte, relaciones amorosas - perdida idealizadora de un ser querido, caracterizado por la aparición de pensamientos, emociones y comportamientos causados por esa pérdida (Guillem Porta, Romero Retes, \& Oliete Ramírez, s.f)

En tanto para la sustentación de la investigación se fundamentó principalmente en la teoría de Kübler-Ross, donde añade que duelo tiene una razón de ser y que si no se pasa por él, sería preocupante puesto que ayuda a evolucionar, madurar y aceptar situaciones nuevas, además de equilibrar lo que la vida se encargó de desequilibrar (Cuadrado, 2010).

La teoría psicoanalítica también fue parte fundamental en el estudio, puesto que Freud en el duelo buscaba trabajar el desapego de las marcas distintivas, en virtud de las cuales el objeto, persona $u$ idea perdida y que estaba integrado a la subjetividad.

Esta investigación pertenece a la línea número uno Salud Mental del área de Humanidades, cuyo objetivo es desarrollar investigaciones que promuevan el abordaje de problemáticas psicosociales relacionados con la Salud Mental, en este caso la experiencia de duelo en cuidadores de personas con parálisis cerebral.

Respecto a los estudios previos referidos al tema, Torrez (2018) en su estudio Experiencias de madres con niños con parálisis cerebral infantil que acuden al control de crecimientos y desarrollo en el C.S. José Oyala, Chiclayo, encontró que todas las madres mencionaron que sus experiencias han sido un proceso de mucho aprendizaje, sufrimientos, cambios y búsquedas por tener una mejora de calidad de vida dentro de todo esto, aunque suene irónico, ha sido una bendición poder contar con esos niños dentro de sus familias. 
Una tesina realizada por Cornejo Aguiar (2008) de la Asociación Mexicana de Tanatología, que lleva por nombre El duelo de los padres por el nacimiento de un hijo con síndrome de Down y parálisis cerebral, tiene como objetivo de investigación ayudar en el conocimiento en cuanto al duelo y en el proceso de adaptación por el que tienen que pasar los padres de familia ante la noticia y consecuencias a raíz del diagnóstico de parálisis cerebral y síndrome de Down, además que con el tiempo aprendan a resolver los obstáculos de vida. La investigación de tanatología dio como resultado que el éxito de las personas con discapacidad, no está determinado por el tipo ni el grado de ésta, se relaciona, sobre todo, con el apoyo y las aportaciones que recibió y la forma en que logró integrarse o no a la sociedad.

La Universidad Nacional Pedro Ruiz Gallo en su estudio: Experiencias de madres con niños con parálisis cerebral infantil que acuden al control de crecimientos y desarrollo en el C.S. José Oyala, Chiclayo-2018, Torrez (2018) hizo énfasis en que todas las madres mencionaron que sus experiencias han sido un proceso de mucho aprendizaje, sufrimientos, cambios y búsquedas por tener una mejora de calidad de vida dentro de todo esto, aunque suene irónico, ha sido una bendición poder contar con esos niños dentro de sus familias.

Un estudio de la Universidad Nacional Autónoma de Nicaragua UNANLEON, con el nombre Estrés y afrontamiento en padres y/o tutores de niños con parálisis cerebral infantil que asisten al centro los pipitos de la ciudad de León, tenía como objetivo conocer las estrategias de afrontamiento que estos padres utilizan, así como también identificar la presencia de estrés en los mismos, obteniendo como resultados que: los padres y/o tutores de niños con parálisis cerebral infantil que asisten al centro los pipitos de la ciudad de León presentan un $46.7 \%$ nivel alto de estrés siendo estos más frecuentes en padres y/o tutores solteros (Martínez \& Reyes, 2009).

Afectaciones psicológicas en padres y madres de hijos(as) con parálisis cerebral infantil que asisten a la fundación Sielo, segundo semestre 2015 es un estudio llevado a cabo por la prestigiosa Universidad Autónoma de Nicaragua UNAN-Managua con el objeto de analizar las afectaciones psicológicas padres de familia de hijos con parálisis cerebral infantil que asisten a la Fundación SIELO. El estudio es descriptivo-analítico de corte transversal, cuya muestra fue de 45 padres, 9 padres de León y 36 padres de Chinandega. Para cumplir con el objetivo que se plantearon, utilizaron el instrumento inventario de estrategias de afrontamiento, obteniendo como resultados que: Los padres en su mayoría por ser mujeres, de religión católica, que cohabitan en unión libre, con nivel de escolaridad secundaria y edades comprendidas entre 26 y 38 años. (Dávila Vanegas \& Rocha Castillo, 2016).

Tomando en cuenta los estudios realizados anteriormente, este estudio puede suministrar datos e información amplia y específica sobre experiencias de duelo, dificultades emocionales, temores y afrontamiento que pasan las 
cuidadoras de personas con diagnóstico de parálisis cerebral, además, dejar un importante aporte al centro de asociación de padres de familia con hijos discapacidad en Los Pipitos Matagalpa, interesados en conocer e implementar nuevos aportes y estrategias para el cuido del entorno familiar de las personas afiliadas al centro, además, de servir para otras investigaciones en el área de Psicología.

La presente investigación se realizó, con el principal objetivo conocer experiencias duelo en cuidadoras de personas con diagnóstico de parálisis cerebral en el centro, Los Pipitos Matagalpa agosto-diciembre 2020, se plantea ya que, desde la concepción psicológica, existe un proceso de duelo en cada uno de los integrantes del entorno de la persona con parálisis cerebral.

\section{MATERIALES Y MÉTODOS}

El presente estudio pertenece al paradigma interpretativo, puesto que no pretende hacer generalizaciones a partir de los resultados obtenidos. Su propósito culmina en la elaboración de una descripción ideográfica de éste, en términos de las características que lo identifican y lo individualizan.

Busca la objetividad en el ámbito de los significados. Se centra en la descripción de lo que es único y personal en el sujeto y no en lo generalizable; pretende desarrollar conocimiento ideográfico y acepta que la realidad es dinámica, múltiple y holística; cuestiona la existencia de una realidad externa valiosa para ser analizada. El objetivo fue comprender la conducta, actos y pensamientos de las personas estudiadas, en este caso cuidadores de personas con diagnóstico de Parálisis Cerebral (en adelante PC). (Rivera, 2010, pág. 6).

La investigación es cualitativa, que es un método de estudio que tiene como objetivo comprender, ponderar e interpretar información obtenida, a través de recursos como entrevistas, conversaciones, registros, memorias, entre otros, con el propósito de indagar en su significado profundo). Además, de ser un estudio cualitativo, un diseño fenomenológico, este se fundamenta en el estudio de las experiencias de vida, respecto de un suceso, desde la perspectiva del sujeto (Fuster Guillen, 2019). Este estudio es de corte transversal, se realizó en un tiempo determinado, abarcando meses agostodiciembre 2020.

El estudio se llevó cabo en el centro Los Pipitos, de la ciudad de Matagalpa ubicado exactamente de la Escuela pública María Cerna Vega una cuadra al sur. En la actualidad en la organización están afiliados 60 niños, niñas jóvenes y adolescentes con diferentes discapacidades, de ellos 12 pacientes con parálisis cerebral. 
La investigación se desarrolló con cuidadoras de personas con parálisis cerebral que asisten al centro Los Pipitos en la ciudad de Matagalpa, con un universo de 12 personas que se dedican al cuido de pacientes con PC. Para este estudio en mención la muestra se representó por cuatro cuidadoras de personas con parálisis cerebral en distintas edades de su vida menores de 40 años. Se trata de una muestra por conveniencia; este muestreo pertenece a los estudios no probabilísticos y permite seleccionar aquellos casos accesibles que acepten ser incluidos (Otzen \& Manterola, 2017).

Criterios que se tomaron en cuenta para la selección de la muestra:

- Cuidadores (mamá, papá, abuelos, hermanos, tíos) de una persona con parálisis cerebral.

- Estar afiliados a la organización Los Pipitos Matagalpa: Los Pipitos llevan más de 20 años funcionando en esta ciudad.

- Ser de la ciudad de Matagalpa: la muestra debe ser exclusivamente de Matagalpa y sus municipios.

- Accesibilidad a brindar información: los cuidadores deben de estar abiertos a contribuir a la investigación.

- Firma de consentimiento informado de participantes.

Los instrumentos seleccionados para la recolección de datos fueron la entrevista en profundidad a las cuidadoras, mismo que permitió la recopilación de información importante sobre las personas estudiadas, una entrevista semiestructurada a una psicóloga que trabaja en la salud mental, la cual permitió obtener datos de relevancia y las posibles técnicas psicológicas para el plan estratégico, además de una guía de observación a las cuidadoras con el objetivo de conocer las vivencias de duelo en cuidadores de personas con diagnóstico de parálisis cerebral.

Para el procesamiento y análisis de datos, primero se realizó la transcripción fiel de la entrevista de acuerdo al dialogo del investigado, además de un análisis de contenido temático, que consiste en un conjunto de métodos y técnicas de investigación destinados a facilitar la descripción e interpretación sistemática de los componentes semánticos y formales de todo tipo de mensaje.

Además, se realizó el sistema de categorías para el análisis y procesamiento de datos con el que se trabajó una tabla que contenía, pregunta de investigación, objetivo específico, categorías, definición conceptual, subcategorías o categorías no contempladas en la investigación, técnicas y recolección de datos, fuentes de información procesamiento y análisis de datos, posteriormente se realizó una transcripción ,análisis y discusión de las tres categorías principales, donde se estudió el aporte fiel de las cuidadoras y subcategorías o categorías emergentes en la investigación. 
También, se trabajó con base a una triangulación de resultados con los instrumentos aplicados para encontrar similitudes en las vivencias de duelo en las cuidadoras, como objetivo central de la investigación. Para la realización de la investigación se trabajó en tres fases, la primera que fue la preparatoria, donde se escogió el tema de investigación, identificación del problema y planteamiento de cuestiones de investigación, se definió y describió la temática abordar. Se hizo revisión de literatura y se redactó antecedentes y objetivos, seguidamente se prosiguió a la búsqueda de participantes y por último se elaboró el marco teórico.

En cuanto a la fase de trabajo de campo: En esta etapa se procedió a la validación de los instrumentos realizados por los investigadores, posteriormente se aplicaron las entrevistas en profundidad, semi estructurada, y la guía de observación y luego se realizó la fase analítica, donde se procesaron los datos obtenidos en los instrumentos y se procedió a la discusión de los mismos para culminar con las recomendaciones y conclusiones del estudio.

Para la ejecución de la investigación fue necesaria la comunicación entre investigador investigado, con el objetivo de brindar información sobre el estudio, cómo serían utilizados los datos obtenidos en los instrumentos. En tanto, los participantes del estudio se plasmaron en la investigación de forma anónima y codificada de la siguiente manera: C1LD, C2A, C3P y C4LY

A los participantes del estudio antes de empezar con la aplicación de instrumentos, se leyó en voz alta el consentimiento informado, donde se les explico que el uso exclusivo de dicha información seria para uso meramente investigativo.

\section{RESULTADOS Y DISCUSIÓN}

La caracterización de las personas que participaron en el estudio son las siguientes:

Tabla 1. Caracterización de sujetos participantes

\begin{tabular}{|c|c|c|c|c|c|c|c|c|}
\hline Cuidadora & Edad & Procedencia & $\begin{array}{l}\text { Estado } \\
\text { civil }\end{array}$ & Parentesco & $\begin{array}{c}\text { Nivel } \\
\text { académico }\end{array}$ & $\begin{array}{l}N^{\circ} \text { de } \\
\text { hijos }\end{array}$ & Ocupación & $\begin{array}{c}\text { Tiempo } \\
\text { de } \\
\text { cuido }\end{array}$ \\
\hline C1LD & 54 & $\begin{array}{l}\text { Matagalpa, } \\
\text { zona } \\
\text { periférica }\end{array}$ & Soltera & Madre & Ninguno & 3 & $\begin{array}{l}\text { Ama de } \\
\text { casa }\end{array}$ & 11 \\
\hline $\mathrm{C} 2 \mathrm{~A}$ & 37 & $\begin{array}{l}\text { Matagalpa, } \\
\text { zona urbana }\end{array}$ & $\begin{array}{l}\text { Unión de } \\
\text { hecho } \\
\text { estable }\end{array}$ & Madre & Universitaria & 1 & $\begin{array}{l}\text { Ama de } \\
\text { casa }\end{array}$ & 10 \\
\hline C3P & 67 & $\begin{array}{l}\text { Matagalpa, } \\
\text { zona urbana }\end{array}$ & Casada & Madre & Alfabetizada & 10 & $\begin{array}{l}\text { Ama de } \\
\text { casa }\end{array}$ & 36 \\
\hline C4LY & & $\begin{array}{l}\text { Matagalpa, } \\
\text { zona urbana }\end{array}$ & Casada & Tía & $\begin{array}{l}\text { Sexto grado } \\
\text { primaria }\end{array}$ & 2 & $\begin{array}{l}\text { Ama de } \\
\text { casa }\end{array}$ & 6 \\
\hline
\end{tabular}




\section{Vivencias del proceso de duelo ante el diagnóstico de parálisis cerebral}

Frecuentemente, la respuesta de la solicitud de participar en un diagnóstico de discapacidad como es la parálisis cerebral, genera en gran manera un shock emocional en las personas cuidadores, ocasionado en algunos casos desesperación, incertidumbre y hasta algunas veces mucho sufrimiento. Esto se observa en el relato de C3P, que le tocó cuidar a dos de sus diez hijos, los cuales fueron diagnosticados con parálisis cerebral.

"Sufrí mucho porque yo también no solo ella la tenía con esa enfermedad, en veces salía descalza, para el hospital porque es muy duro, expreso una de las cuidadoras con lágrimas en sus ojos y quien lleva más de 30 años al cuido de su hija." (Madre 3, cuidadora)

No obstante, es importante remarcar que cada experiencia de duelo ante la pérdida idealizada de su familiar tras el diagnóstico de PC es distinta, así remarcaron las cuidadoras (C2A).

"Terrible porque cuando uno como mujer está embarazada o sea uno piensa que su hijo nazca bien, sin ningún problema, eh... Tenes muchos planes para ese niño, ya... y saber que, hay una lesión y que te cambia total la vida ya, de ese momento que sabes que tu hijo". (Madre 2, cuidadora)

En cuanto los hallazgos encontrados en este estudio y en relación con la investigación realizado por Dávila y Rocha (2016) se coincide que el proceso puede tener diversas formas de representaciones emocionales y entre los más comunes se encuentran la tristeza, enojo, frustración y el dolor, sin embargo, si se difiere en cuanto a que los cuidadores no presentaron sentimientos de rechazo por sus hijos, sobrinos o familiares. En este sentido, Cornejo Aguiar (2008) también, hace referencia a los diferentes sentimientos que se viven al momento de que las personas a cargo de otra con una discapacidad sienten al momento de recibir la noticia de parálisis cerebral u otra afectación.

De acuerdo con los tipos de duelo descritos por Meza Dávalos y otros (2008) en relación con las experiencias de los cuidadores se puntualiza que predomina el duelo resuelto, sin embargo, una de las cuidadoras se encuentra en un duelo crónico que hace referencia a un proceso que puede afectar toda su vida.

En relación al párrafo anterior Torrez (2018) investigación ejecutada en Perú, cita puntualmente: "las emociones que son parte de las vivencias a las que se enfrentan los cuidadores", coincidiendo así con los resultados de este estudio. 


\section{Modificación de la vida cotidiana de cuidadoras de personas con PC}

El cambio de vida después de enfrentarse al nuevo reto de cuidar a una persona con pc, es sin duda una situación de estrés y un cambio radical para la persona que tiene el deber de contribuir a subsistir y ayudar a un paciente con pc. Según los cuidadores, pese a la estigmatización social, discriminación y en la mayoría de los casos carencias económicas han logrado salir adelante, en este sentido C1LD expresó: "Una parte pues uno se siente que cambia, pero pues otra parte normal tranquila gracias a Dios con mi niña, es una bendición de Dios... Yo me siento feliz con ella, aunque con dificultades pues. La idea de esperar un bebe sin ningún problema da un giro al querer sacar adelante a su hijo y ser la prioridad sobre cualquier cosa" (Madre 1, cuidadora).

La situación de tener un familiar cercano con esta patología genera grandes trasformaciones en cada cuidador ya que por su condición ya no pueden trabajar, aun teniendo carreras universitarias, pues tienen que estar casi o a tiempo completo a cargo de la persona con pc situación que da como consecuencia, según sus vivencias dificultades económicas, déficit en las relaciones sociales y muchas veces el corte definitivo de relaciones sentimentales.

En cuanto a la relación de la categoría; Modificación de la vida cotidiana de cuidadores de personas con PC, en el estudio realizado por Torrez (2018) coincido que en el ámbito laboral se producen diversas modificaciones donde en ocasiones la madre deja de trabajar para dedicarse de manera exclusiva al niño, o reduce su jornada de trabajo. En este caso, los resultados arrojaron que las cuidadoras son las que se encargan del cuido especial de las personas con parálisis cerebral.

En cuanto al ámbito social Torrez (2018), refiere el trato de la sociedad es su mayoría es discriminatoria, además de que pese a lo que se viven como madre de hijas con discapacidad, encuentran consuelo en otras madres que comparten su mismo sufrimiento, en este sentido, el estudio realizado por los autores Dávila Vanegas y Rocha Castillo (2016) coinciden con esta subcategoría dado que algunas cuidadoras pueden llegar a establecer relaciones iguales a las de otras cuidadoras, estas amistades sirven para afianzar conocimientos en grupos de autoayuda.

\section{Repercusiones psicológicas del duelo en cuidadores de personas con parálisis cerebral}

La necesidad de sobrellevar la situación hace que los cuidadores busquen refugio y alternativas de ayuda, ya sea en la familia o en organizaciones sociales. En este sentido, las personas que se dedican en su mayoría a ser cuidadores traen consigo una carga emocional extra, pues las patologías subyacentes de los individuos al cuido, hace que el estrés sea mayor en 
momentos de angustia, es decir, cuando la persona con PC se complica por otras enfermedades provocadas por la discapacidad.

"Yo tengo un hïo en el reparto que también me ayuda, pues poco, pero me ayuda y así también doña Yadira (Dra. Los Pipitos) que cuánto hay algo de donación pues ella me apoya también, y yo le agradezco a ella y a Dios, ella nunca me abandona" (Madre 1, cuidadora)

El estar al tanto de una persona con PC es sin duda una experiencia difícil, pero a la vez reconfortante, cada avance que se da cuando se tiene esta patología es impresionante y con gran impacto emocional para los padres, tíos, o tías cuidadores.

"Claro... yo pienso que esto, esto va a cambiar, porque yo siempre le pido a mi Dios todas las noches que algún día pueda verla caminar, pueda verla cambiar que... ya no este solo ahí en la cama, que ya pueda sentarse, que ella también pueda caminar, en mi imaginación" (Madre 3, cuidadora)

El miedo a morir y dejar a la persona con parálisis cerebral es una de los pensamientos que día a día aquejan a la mayoría de las cuidadoras investigadas, pues no conciben dejar a esa persona con cualquier persona o entidad, por miedo a cómo van hacer tratadas. "Si morirme yo y que quede él es la preocupación más grande, a veces cuando estoy enferma yo digo hay pobre mi hijo que va hacer el niño si la que lo alimenta soy yo, aparte de yo que lo alimente la única que me le puede dar comida es mi hermana".

La investigación realizada por Cornejo Aguiar (2008), coincidió que las personas que son cuidadores de otras con PC han buscado ayuda en grupos de auto ayuda como Los Pipitos, en el caso de Martínez, Reyes y Blanco (2009) en Fundación Sielo en Managua y en el caso del estudio de Castillo y Rocha (2016) donde las personas ya sean madres o padres buscaron ayuda en Los Pipitos en la ciudad de León.

Sin bien es cierto, Cornejo Aguiar (2008) hace referencia a que las personas buscan ayuda para superar una perdida, en el caso de ese estudio los sujetos van al tanatólogo, no obstante, en comparación con este estudio los cuidadores buscan al psicólogo o bien alguna institución social, para sobrellevar la situación. Difiriendo de esa manera en ese resultado, aunque se reconoce el papel importante de ese trabajo.

\section{La visión de los expertos}

La experta consultada explicó que el hecho de cuidar a una persona con discapacidad es una situación que toma desprevenida al círculo más cercano del afectado, desde un shock emocional, llanto, conmoción y en algunos casos la negación y sentimientos de culpa. "A la hora del nacimiento todo padre está preparado para recibir un hijo perfecto, con todas las condiciones están 
preparados para recibir un niño que no tenga ninguna deficiencia ninguna dificultad, esperan un desarrollo adecuado y al tener al recibir la noticia pues de que el niño tiene alguna dificultad, es un impacto emocional bien grande el que papá o la mamá vive". Subraya enfática la especialista, aunque advierte que el duelo por parte de la cuidadora o cuidador es distinto, ya sea por el nivel educativo, social o de conocimiento al tema, aunque en los casos que como psicóloga del centro Los Pipitos en Matagalpa, ha atendido, han sido para los cuidadores muy traumático.

Cada caso y situación es distinta, no obstante, afortunadamente los tiempos han cambiado, hay más inclusión y existen esfuerzos mayores de las entidades estatales para integrar a los niños con discapacidad. Los casos de Parálisis Cerebral siguen siendo estigmatizados y discriminados, sin embargo, no se puede quitar el esfuerzo de organizaciones y fundaciones que velan por la integridad de los infantes o adultos que tienen esta patología; "Hace años atrás los padres estaban totalmente en desconocimiento de lo que era una parálisis cerebral, entonces a ellos se les hacia un poco más difícil a enfrentar la situación, pero en la actualidad algunos padres vos sabes que hay mucha curiosidad, hay mucha información, entonces algunos padres mantienen checando ciertas páginas y tienen un poco de información, pero aunque tengan la información un poco no es la adecuada porque no es lo mismo vivirla a tener una información a estar experimentando en si la situación" .

En relación a la teoría de Elizabeth Kübler Ross (Martin Canovas, 2019) y los resultados del presente estudio, la especialista se refiere sobre las cinco etapas del duelo, donde las cuidadoras expresaron que al principio o al momento de la noticia de PC no podían creer, o se negaban a aceptar la situación, esta etapa la psiquiatra la define como Negación. En consiguiente, se encuentra la Ira, donde los cuidadores sienten dolor, tristeza, resentimiento y en algunos casos las personas que dedican al cuido de otra con PC se cuestionan el ¿̇Por qué a mí? La negociación, depresión y aceptación es la última fase que Kübler Ross define en su modelo planteado.

Para Roos (Martin Canovas, 2019), el proceso de adaptación que se vive con el duelo es como un encontronazo que cambia la vida del cuidador, donde el proceso es diferente para cada integrante de la familia de cuya persona diagnosticada. La psiquiatra menciona cinco etapas sobre el proceso de duelo hasta llegar al bienestar, como se mencionaba anteriormente todo proceso es distinto y no todos los cuidadores alcanzaran el bienestar de inmediato en comparación otros, pues les tardara más tiempo el sentir con menos intensidad los síntomas de las etapas anteriormente citadas.

Tomando como referencia lo encontrado en la Madre 3, cuidadora (C3P) quien lleva más de 34 años a cargo de su hija y que al momento de la entrevista en profundidad empezó a llorar por su experiencia de vida, argumentaba lo difícil que ha sido sobrellevar tantos años el cuido de su familiar. En consiguiente, es claro y según el modelo de Elizabeth Kübler en 
dicha cuidadora existe un duelo no culminado, pues no ha llegado a la etapa de la aceptación.

\section{Las repercusiones psicológicas en las cuidadoras}

La guía de observación se utilizó para constatar la existencia de repercusiones psicológicas. Las cuidadoras se encuentran entre los 37 años a los 67 años. En cuanto a las observaciones se realizaron en sus domicilios, acordando con anticipación día y la hora disponible a su persona, además se visualizó disposición y amabilidad al recibir al equipo de investigadores en sus hogares. Todas las cuidadoras estaban coherentes en tiempo, espacio y persona.

Se percibió que la mayoría de las cuidadoras sufren el apego con sus familiares sin dejar que alguien más se encargue del cuidado, argumentando que solo ellas saben cómo darles de comer, bañarlos y como comunicarse. En cuanto a su expresividad y argumentación, se observó congruencia en cada uno de los relatos. En consiguiente, con la categoría número uno se observó la aparición de distintos sentimientos al momento de la noticia de discapacidad, esto al rememorar su vivencia como cuidadores de personas con PC.

De acuerdo con el apego emocional evidenciado en la categoría número tres sobre repercusiones psicológicas, y en base a la literatura científica investigado, este apego forma parte fundamental en la vida de los cuidadores, pues el cariño y el amor siempre son constante, donde también este sentimiento forma parte de un mecanismo de defensa para evitar la culpabilidad de su sentir como persona Castillo \& Rocha (2016).

\section{La propuesta de un plan de intervención general}

En este estudio se propone un plan de intervención, donde se detallan estrategias y técnicas psicológicas, para contrarrestar, estrés, ansiedad, sentimientos de culpas entre otras. Además de brindar sugerencias de talleres sobre cuido personal y motivación. 
Tabla 2. Plan de intervención general

\begin{tabular}{|c|c|c|c|c|}
\hline Objetivo & $\begin{array}{l}\text { Modalidad } \\
\text { de } \\
\text { intervención }\end{array}$ & Propósito & $\begin{array}{l}\text { Técnicas de } \\
\text { intervención }\end{array}$ & Contenido a trabajar \\
\hline \multirow[t]{2}{*}{$\begin{array}{l}\text { Reducir el nivel } \\
\text { de estrés en } \\
\text { los cuidadores }\end{array}$} & \multirow[t]{2}{*}{$\begin{array}{l}\text { Individual } \\
\text { (cuidador) }\end{array}$} & \multirow[t]{2}{*}{$\begin{array}{l}\text { Individual: } \\
\text { que hace } \\
\text { referencia al } \\
\text { encuentro entre } \\
\text { el terapeuta } \\
\text { y el paciente, } \\
\text { donde se crea } \\
\text { un ambiente } \\
\text { de aceptación, } \\
\text { confidencialidad } \\
\text { y apertura el } \\
\text { paciente puede } \\
\text { expresar sus } \\
\text { problemas y } \\
\text { emociones. }\end{array}$} & $\begin{array}{l}\text { Terapia de } \\
\text { relajación }\end{array}$ & $\begin{array}{l}\text { Relajación muscular: es } \\
\text { un método de relajación } \\
\text { progresiva. } \\
\text { Comienzas por relajar y } \\
\text { tensar los músculos de los } \\
\text { dedos de los pies, se continua } \\
\text { gradualmente hacia arriba } \\
\text { hacia el cuello y cabeza. } \\
\text { También puede comenzar } \\
\text { por la cabeza y el cuello y } \\
\text { continuar el trabajo hacia } \\
\text { abajo, hacia los dedos de los } \\
\text { pies. Contrae los músculos } \\
\text { durante unos cinco segundos } \\
\text { y luego relájelos durante unos } \\
30 \text { segundos y repite. }\end{array}$ \\
\hline & & & Visualización & $\begin{array}{l}\text { Para relajarte con la } \\
\text { visualización, intenta } \\
\text { incorporar tantos sentidos } \\
\text { como puedas como el olfato, } \\
\text { la vista el oído y el tacto. } \\
\text { Por ejemplo, el océano } \\
\text { imagínate el sonido de las olas } \\
\text { golpeando la orilla, la calidez } \\
\text { del sol sobre tu cuerpo, el } \\
\text { caminar bajo la arena. } \\
\text { Es recomendable cerrar los } \\
\text { ojos, sentarte en un lugar } \\
\text { tranquilo, usar ropa cómoda y } \\
\text { concentrarse en la respiración, } \\
\text { intentar concentrarse en el } \\
\text { presente y pensamientos } \\
\text { positivos. }\end{array}$ \\
\hline
\end{tabular}




\begin{tabular}{|c|c|c|c|c|}
\hline Objetivo & $\begin{array}{c}\text { Modalidad } \\
\text { de } \\
\text { intervención }\end{array}$ & Propósito & $\begin{array}{l}\text { Técnicas de } \\
\text { intervención }\end{array}$ & Contenido a trabajar \\
\hline \multirow[t]{3}{*}{$\begin{array}{l}\text { Sensibilizar a } \\
\text { los cuidadores } \\
\text { acerca de la } \\
\text { organización } \\
\text { de su tiempo } \\
\text { y fomentar } \\
\text { estrategias } \\
\text { de auto } \\
\text { cuido para la } \\
\text { prevención del } \\
\text { desgaste en } \\
\text { cuidadores }\end{array}$} & \multirow[t]{3}{*}{$\begin{array}{l}\text { Grupal } \\
\text { (cuidadores) }\end{array}$} & \multirow[t]{3}{*}{$\begin{array}{l}\text { Grupal: se } \\
\text { desarrolla en } \\
\text { grupos de } \\
\text { autoayuda } \\
\text { y se brindan } \\
\text { pautas para } \\
\text { afrontamiento } \\
\text { de sus } \\
\text { sentimientos. }\end{array}$} & $\begin{array}{l}\text { Talleres: } \\
\text { Taller de auto } \\
\text { cuido } \\
\text { Taller de } \\
\text { cuídate para } \\
\text { cuidar }\end{array}$ & $\begin{array}{l}\text { El auto cuido tiene como } \\
\text { finalidad que los cuidadores } \\
\text { conozcan, compartan } \\
\text { experiencias sobre sus vidas y } \\
\text { que en conjunto desarrollen el } \\
\text { concepto de auto cuido y vida } \\
\text { sana, comprometiéndose a } \\
\text { practicar alguna actividad que } \\
\text { fomente esta forma de vida. }\end{array}$ \\
\hline & & & $\begin{array}{l}\text { Talleres de } \\
\text { motivación }\end{array}$ & $\begin{array}{l}\text { Taller de Motivación: se } \\
\text { empleará una parte teórica } \\
\text { con una metodología } \\
\text { participativa en el que se } \\
\text { pondrán en práctica ejercicios } \\
\text { y distintas técnicas que } \\
\text { afiancen aprendizaje. La } \\
\text { herramienta fundamental del } \\
\text { taller es el uso de la psicología } \\
\text { positiva para conseguir un } \\
\text { cambio en la persona. }\end{array}$ \\
\hline & & & $\begin{array}{l}\text { Técnicas que } \\
\text { ayuden a } \\
\text { organizar el } \\
\text { tiempo de } \\
\text { cuido }\end{array}$ & $\begin{array}{l}\text { Técnicas de organización de } \\
\text { tiempo: } \\
\text { - División de } \\
\text { responsabilidades de los } \\
\text { cuidadores } \\
\text { - Tiempo en familia } \\
\text { - Vida social } \\
\text { - Vida laboral }\end{array}$ \\
\hline $\begin{array}{l}\text { Fortalecer } \\
\text { proyectos de } \\
\text { vida y lazos } \\
\text { sentimentales } \\
\text { en el } \\
\text { matrimonio y } \\
\text { la familia }\end{array}$ & $\begin{array}{l}\text { De pareia y } \\
\text { de familia }\end{array}$ & $\begin{array}{l}\text { De pareja y } \\
\text { familiar: se } \\
\text { refiere a una } \\
\text { terapia donde la } \\
\text { pareja o familia } \\
\text { pueda expresar } \\
\text { su sentir ante la } \\
\text { situación y donde } \\
\text { se proponen } \\
\text { estrategias y } \\
\text { percepción ante } \\
\text { la situación } \\
\text { vivida. }\end{array}$ & $\begin{array}{l}\text { Técnicas: } \\
\text { Crea tu } \\
\text { proyecto de } \\
\text { vida } \\
\text { Taller de plan } \\
\text { de vida }\end{array}$ & $\begin{array}{l}\text { Fortalecimiento de la relación } \\
\text { de pareja estableciendo } \\
\text { confianza y la comunicación } \\
\text { de la relación }\end{array}$ \\
\hline
\end{tabular}




\section{CONCLUSIONES}

De acuerdo con el análisis y discusión de resultados las cuidadoras de personas de parálisis cerebral al momento de recibir la noticia de que su hijo, hija, sobrino o la persona a su cargo, tendrá PC, es una situación que les genera un shock emocional fuerte, pues el diagnóstico que los médicos les proveen, nunca se lo esperaron, dando como resultado sentimientos de culpa, tristeza, llanto y hasta rabia por la condición de los cuidados. Sin duda, el diagnóstico de esta condición genera que los cuidadores desechen la idealización de un hijo sin discapacidad que con anterioridad se habían formulado.

El hecho de no saber de qué trataba la parálisis cerebral fue un factor muy importante pues genero sentimientos de desesperanza, impotencia, negación e incluso reproche hacia los mismos cuidadores. No obstante, la ayuda de su familia fue transcendental para su nueva realidad.

El cambio de vida es un factor que tomó por sorpresa a los cuidadores, donde se tuvieron que adaptar a la nueva vida y circunstancias que la condición de la persona con PC ofrecía. El cambio de vida es un factor que tomo por sorpresa a los cuidadores, donde se tuvieron que adaptar a la nueva vida y circunstancias que la condición de la persona con PC ofrecía. En cuanto a la sociedad en términos de inclusión todas las cuidadoras consultadas externaron que aún la población nicaragüense no está realmente concienciada sobre la aceptación y empatía en términos de discapacidad, de igual manera sus relaciones sociales fueron disminuyendo por el tiempo de demanda que exigen las personas con parálisis cerebral.

El apoyo familiar es de vital importancia no solo para la persona con parálisis cerebral, sino también para la cuidadora, de acuerdo con el estudio ejecutado las cuidadoras sintieron el cambio abrupto en su economía desde la llegada de la persona con PC.

En consiguiente, se concluye que una de las cuatro cuidadoras participes del estudio realizado, todavía sigue viviendo un proceso de duelo doloroso y que, aunque los años han pasado la vivencia del diario vivir, le lastima y todavía se cuestiona el porqué de la situación y según la consulta a diversas bibliografías hace referencia a un duelo no resuelto o crónico, puesto que lleva más de tres décadas desde el nacimiento de su hija.

En cuanto a los resultados la presente investigación se ofrece un plan de intervención, que fue elaborado de acuerdo con las vivencias de los cuidadores, expertos e investigadores, se propone un programa con diversas estrategias metodológicas y donde el plan de intervención se basa en tres 
ejes fundamentales: plan individual, familiar, es decir la pareja y circulo sanguíneo familiar del cuidador y por último un plan de intervención grupal que abarca los grupos de autoayuda del Centro Los Pipitos en Matagalpa.

\section{REFERENCIAS BIBLIOGRÁFICAS}

Castillo Davila, E., \& Rocha, B. (2016). Afectaciones psicologicas en padres y madres de hijos (as) con paralisis cerebral infantil que asisten a a fundación Sielo, segundo semestre 2015. Managua, Nicaragua: UNAN-Managua.

Cornejo Aguiar, C. P. (2008). El duelo de los padres por el nacimiento de un hijo con Sindrome Down y paralisis cerebral. Asociación Mexicana de Educación Continua y a Distancia, A.C., México. Obtenido de http:// www.tanatologia-amtac.com/descargas/tesinas/04\%20El\%20dvelo\%20 de\%20los\%20padres.pdf

Cuadrado, D. (Marzo de 2010). Las cinco etapas del cambio. Capital Humano(241), 54-58. Obtenido de https://factorhuma.org/attachments_ secure/article/420/c303_cincoetapas.pdf

Dávila Vanegas, E. d., \& Rocha Castillo, B. S. (2016). Afectaciones psicologicas en padres y madres de hijos/as con paralisis cerebral infantil que asisten a la fundación SIELO, II semestre 2015. UNAN-Managua, Departamento de Psicología. Managua, Nicaragua: UNAN-Managua. Obtenido de https:// repositorio.unan.edu.ni/10380/1/9143.pdf

Dupas, M. R. (2013). Experiencias de familias de niños con parálisis cerebral en contexto de vulnerabilidad social. Latino-Am. Enfermagem.

Fuster Guillen, D. E. (2019). Investigación cualitativa: Método fenomenológico hermenéutico. (U. N. Marcos, Ed.) Propósitos y Representaciones, 7(1), 201-229 . Obtenido de http://www.scielo.org.pe/scielo.php? script=sci_ arttext\&pid $=$ S2307-79992019000100010

Guillem Porta, V., Romero Retes, R., \& Oliete Ramírez, E. (s.f). Manifestaciones de duelo. Manual. (I. V. (IVO), Ed.) Valencia, Valencia, España: Instituto Valenciano de Oncología (IVO). Obtenido de https://seom.org/seomcms/ images/stories/recursos/sociosyprofs/documentacion/manuales/duelo/ duelo08.pdf

Madrigal, A. (2004). Paralisis Cerebral (Vol. 1). (I. d. (IMSERSO), Ed.) España, Castilla y León, España: Observatorio de la Discapacidad. Obtenido de https://sid-inico.usal.es/idocs/F8/FDO8993/paralisis_cerebral.pdf

Martin Canovas, E. (22 de Enero de 2019). Centro de Psicología Integral. Recuperado el 2021, de https://centrodepsicologiaintegral.com/las-5fases-o-etapas-del-duelo-la-teoria-de-kubler-ross/

Martinez Blandón, M. C., Reyes Saavedra, D. E., \& Blanco Rodriguez, S. M. (2009). Estrés y afrontamiento en padres y/o tutores de niños con parálisis cerebral que asisten al centro "Los pipitos" - León y al CRRETChinandega. León, Nicaragua, León, Nicaragua: Universidad Autonoma 
de Nicaragua - León. Obtenido de http://riul.unanleon.edu.ni:8080/ ispui/bitstream/123456789/2691/1/216170.pdf

Meza Dávalos, E. G., García, S., Torres Gómez, A., Castillo, L., Sauri Suárez, S., \& Martínez Silva, B. (2008). El proceso del duelo. Un mecanismo humano para el manejo de las pérdidas emocionales. (I. d. México, Ed.) Revista de Especialidades Médico-Quirúrgicas, 13(1), 28-31. Obtenido de https://www.redalyc.org/articulo.oa? id =47316103007

Olivares Angeles, F. (2013). Cómo viven las madres el proceso de duelo ante el nacimiento de un hijo con discapacidad. Universidad Autonoma del Estado de México, Psicología. Estado de Mexico: Universidad Autonoma del Estado de Mexico. Obtenido de http://ri.uaemex.mx/bitstream/ handle/20.500.11799/40446/LIC_PSICOLOGIA\%20_Francis_Duelo_ Discapacidad_Madres_13.pdf; sequence $=1$

Otzen, T., \& Manterola, C. (2017). Técnicas de Muestreo sobre una Población a Estudio. International Journal of Morphology(35), 227-232. Obtenido de https://scielo.conicyt.cl/pdf/iimorphol/v35n 1/art37.pdf

Rivera, Y. S. (2010). ¿̇Como se pueden aplicar los distintos paradigmas de la investigación a la cultura física y el deporte? Revista electrónica Ciencia e innovación tecnológica en el deporte(11), 6. Obtenido de https://dialnet. unirioja.es/servlet/articulo? codigo $=6174061$

Torrez, A. (2018). Experiencias de madres con niños con parálisis cerebral infantil que acuden al control de crecimiento y desarrollo c.s. José Olaya, Chiclayo - 2018. Chiclayo, Perú, Perú: Universidad Nacional "Pedro Ruiz Gallo". 